\title{
Nuevos postulados básicos en la contabilidad mundial
}

Raúl A. Arrarte Mera ${ }^{1}$

Recibido: 30 de noviembre 2012

Aprobado: 15 de mayo de 2013

\begin{abstract}
Arrarte R. (2013). Nuevos postulados básicos en la contabilidad mundial. Activos, 20, 157-179
\end{abstract}

\section{Clasificación JEL}

M40, M41

\section{Resumen}

Este trabajo de investigación se preparó especialmente, en el mes de junio de 2013, para el XVIII Congreso Internacional de Contaduría, Administración e Informática a realizarse en la ciudad de México D.F. los días 2 y 3 de octubre de 2013. El objetivo general es examinar la aplicación de los nueve postulados básicos a la luz de los principios de contabilidad tradicionales generalmente aceptados. La investigación desarrollada es analíticoexplicativa-documental y de síntesis bibliográfica de libros, revistas de investigación contable internacionales, compendios y páginas web. La conclusión principal demuestra que son normas de alta calidad, orientadas al inversor; priorizan lo financiero a lo económico; son provenientes del consenso alcanzado por diferentes corrientes teóricas desarrolladas en las

1 Doctor en Ciencias Contables y Empresariales y Contador Público de la Universidad Nacional Mayor de San Marcos. Maestro en Administración de la Universidad de San Martín de Porres. Docente Principal de la Facultad de Ciencias Contables de la UNMSM. Correo electrónico: raularrarte@speedy.com.pe 
últimas décadas, validadas por la práctica de los negocios y cuya finalidad es reflejar la esencia económica de las operaciones, y presentar una imagen fiel de la situación financiera de la empresa. LasNIIFs se emiten por la International Accounting Standards Board, IASB, (anterior International Accounting Standards Committee).

\title{
Palabras clave
}

NIIF, postulados básicos, principios contables

\section{Arrarte R. (2013). New basic postulates in global accounting.} Activos, 20, 157-179

\begin{abstract}
This research was especially prepared in the month of June 2013, for the XVIII International Congress of Accounting, Management and Information Technology to be held in Mexico City DF on the $2^{\text {nd }}$ and $3^{\text {rd }}$ of October of 2013. The overall objective is to examine the implementation of the nine basic postulates in light of the traditional accounting principles generally accepted. The research developed is analytical-explanatory documentary and a bibliographic synthesis of books, international accounting research journals, abstracts and Web pages. The main finding shows that there are high quality standards geared to the investor, prioritizing financial rather than economic concerns, from the consensus reached by different theoretical approaches developed in recent decades, validated by business practice and whose aim is to reflect the economic essence of operations, and to present a fair view of the financial position of the company. IFRS are issued by the International Accounting Standards Board (IASB), (former International Accounting Standards Committee).
\end{abstract}

\section{Keywords}

IFRS, basic postulates, accounting principles 


\section{Arrarte R. (2013). Nouveaux postulats de base de la comptabilité mondiale. Activos, 20, 157-179}

\section{Résumé}

Ce document de recherche a été préparé surtout au mois de juin 2013, pour le $18^{\text {ème }}$ Congrès International de Comptabilité, de Gestion et d'Informatique, qui se tiendra dans la ville de Mexico D. F. le 2 et 3 octobre 2013. L’objectif général est d'examiner l'application des neuf postulats de base, à la lumière des principes de la comptabilité traditionnelle généralement acceptés. La recherche développée est analytique-explicative-documentaire et de synthèse bibliographique de livres, de revues internationales de recherche comptable et de résumés et pages web. La conclusion principale montre que ce sont des normes de haute qualité, orientées vers l'investisseur et qui donnent la priorité à la partie financière et non pas à l'économique. Elles proviennent du consensus des différents courants théoriques développés au cours des dernières décennies, validés par la pratique des affaires et dont le but est de refléter l'essence économique des opérations et de présenter une image fidèle de la situation financière de la société. Les IFRS sont émises par IASB, l'International Accounting Standards Board (ancien International Accounting Standards Committee).

\section{Mots clés:}

IFRS, postulats de base, principes comptables.

\section{Introducción}

Distintos eventos históricos relacionados con la realidad de las empresas originaron cambios en el uso de los sistemas contables a nivel mundial entre los siglos XVIII al XX:

6. La Revolución industrial y su influencia en la contabilidad de costos y la contabilización de la depreciación, 
7. El crecimiento y el desarrollo de los ferrocarriles,

8. La regulación gubernativa de los negocios,

9. La imposición fiscal de las empresas,

10. El desarrollo de los gigantes industriales y financieros mediante fusiones y

11. La influencia de la teoría económica.

Pero, ¿qué milagro ocurrió para generar la unificación de criterios contables entre el Instituto Americano de Contadores y la Bolsa de Valores de Nueva York? (Wikipedia, s.f.)

La llamada Gran Depresión se originó en los Estados Unidos, a partir de la caída de la bolsa del 29 de octubre de 1929 (conocido como Martes Negro, aunque cinco días antes, el 24 de octubre, ya se había producido el Jueves Negro), y rápidamente se extendió a casi todos los países del mundo, debido al sobreprecio de los valores bursátiles, provocando la aparición del movimiento de innovación contable.

Con esta corriente, se cuestionó por primera vez la total libertad de acción que existía en el campo contable, gran responsable por la falta de comparación de la información patrimonial que tenía que ser divulgada por las empresas de la época. Esta dolorosa experiencia coadyuvó a la aparición en el mundo de los Modelos de Estados Financieros que se obliga publicar, trimestralmente, a las sociedades mercantiles, y se exige la presencia del Estado en el control de los Registros Públicos, la emisión de leyes sobre banca y seguros, códigos de comercio, etc.

En palabras de Pérez (2010):

Tras cada debacle financiera, la demanda de cambios en la contabilidad ha sido una constante en la historia del mundo occidental, incluidos los gritos, protestas y demandas de soluciones urgentes y rápidas. Esta vez, tampoco ha sido diferente. Aun cuando nadie bien informado ha culpado nunca a la contabilidad de los desastres económicos, y especialmente los bursátiles, los esfuerzos renovadores tras cada crisis económica han influido en el ritmo 
del cambio contable. El reto esta vez será encontrar unos criterios contables que aborden la complejidad con que hoy en día se desarrolla el mundo de los negocios, y además que sean válidos y aceptados universalmente (p. 45).

¿Qué otros acontecimientos obligaron al cambio de los "criterios tradicionales" a los que ahora aplica en el mundo la International Accounting Standard Board, IASB? Andersen Consulting (2000), sobre la presencia de los mercados de valores tecnológicos, plantea que:

Surgen ante la necesidad de dotar de financiación a empresas de tecnología de punta y con escasa historia de operaciones que, generalmente, no cumplen los requisitos necesarios para cotizar en mercados normales. Estos mercados tienen condiciones de admisión diferentes a las de los mercados tradicionales: se sustituye el requisito de obtención de beneficios por un requisito de fondos propios, ventas o activos y se exigen free floats mínimos (p. 209).

El mercado Nasdaq nació de una iniciativa del Congreso para regular el mercado de acciones Over The Counter (OTC o mercado no organizado). Creado en 1971 como el primer mercado electrónico del mundo, actualmente es el de mayor crecimiento de Estados Unidos y el segundo mayor a escala mundial por capitalización bursátil después del NYSE (Bolsa de Valores de Nueva York). En 1963, la SEC (Comisión de Valores de Nueva York) llega a la conclusión de que el mercado OTC era oscuro y fragmentado y que no contribuía a una creación eficiente de precios. La SEC da el mandato de creación de un mercado automatizado a los intermediarios National Association of Securities Dealers [NASD], y su construcción se inicia en 1968. En 1971 comienza a funcionar bajo la nomenclatura de National Association of Securities Dealers Automated Quotation, NASDAQ, con más de 2.500 valores. Éste será el primer mercado automatizado del mundo, frente a los mercados tradicionales de viva voz.

Pero el colmo de los problemas de información que ya sentían las empresas transnacionales se dio en 1993. "Daimler-Benz (ahora Daimler Chrysler) fue la primera compañía alemana que se inscribió en la Bolsa 
de Valores de Nueva York (NYSE), lo cual sucedió en 1993" (Doupnik, 2007). Anteriormente, las compañías alemanas se habían negado a hacer los ajustes necesarios para reconciliar sus estados financieros, basados en leyes alemanas, con los principios de contabilidad generalmente aceptados de los Estados Unidos de Norteamérica.

Después de algún compromiso con la SEC y debido al fuerte deseo de Daimler por entrar al mercado de capitales estadounidenses (y por ser la primera compañía alemana en hacerlo), Daimler estuvo de acuerdo en cumplir con las regulaciones de la SEC (...) El hecho es que, en 1993, DaimlerBenz reportó una utilidad bajo los principios de contabilidad alemanes, pero una pérdida bajo los principios de contabilidad estadounidenses creó toda una conmoción en la comunidad financiera internacional (...) La cual fue de cerca de DM 2.5 miles de millones (...) En 1996, Daimler-Benz decidió abandonar los principios de contabilidad alemanes e implantar un sistema de contabilidad basado en los principios de contabilidad estadounidenses a nivel mundial (Doupnik, 2007).

El detonante de esta realidad fue que la profesión contable y los organismos mundiales a cargo del establecimiento de normas (llámese Organización de Naciones Unidas, la Unión Europea, la Asociación Internacional de Bolsas de Valores, IOSCO, la Federación Internacional de Contadores, IFAC, dada la importancia de las actividades de la International Accounting Board, IASB, la cual tomó el lugar del IASC en el año 2001), han estado bajo la presión de las compañías multinacionales, de las bolsas de valores, de los reguladores de valores y de instituciones internacionales de préstamos como el Banco Mundial para reducir la diversidad y armonizar los principios y prácticas de contabilidad a nivel internacional.

Es en este ambiente que enmarcamos el desarrollo de este trabajo de investigación y que revisaremos a continuación:

a. Los principios de contabilidad tradicionales.

b. Los principios de la contabilidad mundial.

c. Por qué resulta necesaria la contabilidad mundial. 


\section{Marco de referencia}

\subsection{Principios de Contabilidad Tradicionales}

Estos principios son los siguientes:

- Entidad, señala que la contabilidad está referida a las operaciones de una empresa, la cual es distinta a su dueño o empresarios, por lo cual no corresponde mezclar ambos intereses. Este plano pasa a constituir un principio ético, por el que el Contador deberá cautelar su cumplimiento, evitando, por ejemplo, que los gastos particulares del propietario sean contabilizados como gastos de la empresa.

Principio que pasa a ser un postulado básico, dado que las instituciones fiscales de todo el mundo lo han reglamentado tan minuciosamente, que ya nada queda al azar. Sin embargo, en las Normas Internacionales se le reconoce como "Entidad Económica".

- Empresa en marcha, mediante el cual se presume que no existe un límite de tiempo en la prolongación operacional de la entidad económica.

Este principio se ha mantenido como postulado básico en las Normas Internacionales como "Negocio en Marcha".

- Hechos Económicos, establece que la contabilidad registra solo hechos económicos, de modo que los estados contables se refieren a hechos, recursos y obligaciones económicas susceptibles de ser valorizados en términos monetarios.

Este principio se ha mantenido como postulado básico en las Normas Internacionales como "Sustancia Económica".

- Moneda común denominador, obliga a la contabilidad a medir en términos monetarios, lo cual permite reducir todos sus componentes heterogéneos a un común denominador. 
Este principio es recogido en la NIC No. 21 o Efecto de las Variaciones en los Tipos de Cambio de la moneda extranjera, y como es de suponer, "una entidad puede llevar a cabo actividades en el extranjero de dos maneras diferentes. Puede realizar transacciones en moneda extranjera o bien puede tener negocios en el extranjero. Además, la entidad puede presentar sus estados financieros en una moneda extranjera” (IASB, 2006), para los que define hasta cuatro tipos de moneda de acuerdo con las necesidades empresariales en el mundo global:

Moneda de informe o presentación. Aquella elegida y utilizada por una entidad para presentar sus estados financieros.

Moneda de registro. Es aquella en la cual la entidad mantiene sus registros contables, ya sea para fines legales o de información.

Moneda extranjera. Es cualquier moneda distinta a la de registro, a la funcional o a la de informe de la entidad, según las circunstancias.

Moneda funcional. Aquella con la que opera una entidad en su entorno económico primario; es decir, en el que principalmente genera y aplica efectivo.

- Periodo Contable, considera que la vida de las empresas está dividida en periodos iguales y sucesivos de tiempo, lo cual facilita su control y la evaluación de sus actividades.

Para el caso de Perú, el periodo contable de un año es el ejercicio fiscal que se inicia el $1^{\circ}$ de enero y termina el 31 de diciembre.

Este principio se ha mantenido como postulado básico en las Normas Internacionales como "Asociación de Costos y Gastos con Ingresos".

- Exposición, norma que al término de cada periodo, denominado "ejercicio", se deberá hacer una "exposición" acerca de la situación empresarial mediante la confección de estados contables. 
La NIC No. 1 dice:

"Establecer el marco global para la presentación de estados financieros para uso general, incluyendo lineamientos sobre su estructura y el contenido mínimo.

Principios fundamentales que subyacen a la preparación de estados financieros, incluyendo la hipótesis de empresa en marcha, la uniformidad de la presentación y clasificación, el principio contable del Devengamiento y la importancia relativa.

- Los activos y pasivos, así como los ingresos y gastos, no pueden compensarse salvo que la compensación esté permitida o sea exigida por otra NIIF.

- Debe presentarse información comparativa correspondiente al ejercicio anterior para los importes incluidos en los estados financieros y en la información complementaria.

- Generalmente, los estados financieros deben prepararse con una periodicidad anual. Si cambia la fecha de cierre del ejercicio y los estados financieros se presentan para un periodo distinto al año, será necesario informar este hecho.

- Un conjunto completo de estados financieros debe incluir:

- Un estado de situación financiera.

- Un estado del resultado integral.

- Un estado de cambios en el patrimonio neto.

- Un estado de flujos de efectivo.

- Notas explicativas"(IASB, 2005).

- Devengado, considera que la determinación de los resultados de operación y la posición financiera deben tomar en cuenta todos los recursos y obligaciones del periodo, aunque estos hayan sido o no percibidos o pagados, es decir, debe considerar lo pagado y lo comprometido, con el objeto de que tanto los costos como los gastos puedan ser debidamente relacionados con los respectivos ingresos que generan.

Este principio se ha mantenido como postulado básico en las Normas Internacionales de Información Financiera en el marco de la Contabilidad Mundial, que en adelante se denominará "Devengación Contable". 
- Realización, consiste en determinar los resultados económicos cuando la operación que los origina queda perfeccionada, estableciendo en consecuencia cuándo se debe considerar percibido un ingreso.

Este principio se sustituye en las Normas Internacionales por el postulado básico "Devengación Contable”.

Y se incorpora con un significado más amplio, aun cuando conserva su esencia, con el fin de establecer el momento de reconocimiento de los efectos derivados de las operaciones que lleva a cabo una entidad y de otros eventos que la afectan. Dicho cambio se establece para adecuar la terminología conceptual al entorno internacional, dado que los organismos líderes emplean el término de "devengación contable" o "devengo contable" para establecer que el sistema de información contable opera sobre una base de acumulación más que sobre una base de cobro o pago de efectivo (Comisión de Principios de Contabilidad del Instituto Mexicano de Contadores, 2006a).

- Prudencia, este principio general se puede expresar también diciendo: "Contabilizar todas las pérdidas cuando se conocen y las ganancias solamente cuando se hayan realizado; también nos dice que es cuando el Contador tiene dos o más opciones, pero éste elegirá el que le muestre la cifra menor de dos valores de un activo relativo a una partida determinada". Significa que cuando se deba elegir entre dos valores para un elemento del activo, normalmente se debe optar por el más bajo, o bien que una operación se contabilice de tal modo que la alícuota del propietario sea menor.

Tanto el Consejo de la Financial Accounting Standard Board, FASB, como el General Agreed Accounting Principles of the United States of America, USGAAP, prefirieron no incluir el conservadurismo (la prudencia) dentro del marco conceptual de las normas internacionales porque éste no resultaba útil a los inversores, ya que podía ser manipulado para dar una información ficticia acerca de los estados financieros de las empresas y entrar en conflicto con principios como la fidelidad de representación, la comparabilidad, la coherencia y la neutralidad; lo anterior ocasiona que los preparadores de la información la filtren, quitando así el derecho a los usuarios de decidir qué información es relevante o no (Silva \& Azúa, 2006). 
- Costo como base de valuación, establece que el registro de las operaciones se basa en el valor económico que tienen los bienes y servicios, es decir, el "costo", ya sea de producción, adquisición o canje, el cual actúa como base para la valuación de los mismos.

- El principio de valor histórico original se sustituye por el postulado de valuación, el cual establece la forma de cuantificar las operaciones que lleva a cabo una entidad y otros eventos que la afectan. El postulado de valuación no busca profundizar sobre las posibles bases de valuación de los elementos de los estados financieros (Comisión de Principios de Contabilidad del Instituto Mexicano de Contadores, 2006b).

- Consistencia o Uniformidad, señala que los procedimientos de cuantificación utilizados deben ser uniformemente aplicados de un ejercicio a otro, de modo que cuando existan razones fundadas para cambiar de procedimientos, deberá informarse este hecho y su efecto; esto tiene una gran importancia puesto que uniforma los procedimientos y métodos aplicados, de manera que cuando una operación específica es registrada de una forma determinada, cuando vuelva a acontecer una operación similar en el futuro deberá ser registrada en la misma forma anterior.

Este principio se ha mantenido como postulado básico en las Normas Internacionales de Información Financiera en el marco de la Contabilidad Mundial que en adelante se denominará "Consistencia".

- Materialidad o Importancia Relativa, establece que para imputar las partidas deberá tenerse en cuenta su importancia monetaria y/o vida útil significativa, ya que si, por ejemplo, se adquieren útiles de aseo, debido a que la importancia económica de estos es relativamente baja, a la vez que su vida útil es escasa, no tendría objeto imputarlos como bienes de la empresa, por cuanto serán rápidamente consumidos y pasarán a ser un gasto, por lo tanto, en lugar de ser activados como bienes, son imputados directamente como gastos.

La NIC No. 1 Presentación de Estados Financieros lo redefine de esta manera: 
Materialidad (o importancia relativa). La evaluación acerca de si una omisión o inexactitud puede influir en las decisiones económicas de los usuarios, considerándose así material o con importancia relativa, requiere tener en cuenta las características de tales usuarios. El Marco Conceptual para la Preparación y Presentación de la Información Financiera establece, en el párrafo 25, que: "se supone que los usuarios tienen un conocimiento razonable de las actividades económicas y del mundo de los negocios, así como de su contabilidad, y también la voluntad de estudiar la información con razonable diligencia” (IASB, 2006, p. 54). En consecuencia, la evaluación necesita tener en cuenta cómo puede esperarse que, en términos razonables, usuarios con las características descritas se vean influidos, al tomar decisiones económicas.

- Partida Doble, que se constituye de:

a. Los recursos de los que dispone la entidad para la realización de sus fines, y,

b. Las fuentes de dichos recursos, que a su vez son la especificación de los derechos que sobre los mismos existen considerados en su conjunto.

Este principio se ha mantenido como postulado básico en las Normas Internacionales de Información Financiera en el marco de la Contabilidad Mundial como "Dualidad Económica".

- Equidad, principio fundamental que debe orientar la acción del profesional contable en todo momento y se enuncia así: la equidad entre intereses opuestos debe ser una preocupación constante en contabilidad, puesto que los que sirven de, o utilizan los datos contables, pueden encontrarse ante el hecho de que los intereses particulares se hallen en conflicto.

En las Normas Internacionales de Contabilidad se denomina también "Integridad" y se enuncia de la siguiente manera:

Para ser confiable la información contable contenida en los estados financieros debe ser neutral, es decir, libre de sesgos. Los estados financieros no serán neutrales si, vía selección o presentación de la información, se influencia la toma de decisiones o el juicio a fin de lograr un resultado predeterminado (IASB, 2006, p. 55). 


\subsection{Principios de contabilidad mundial}

Las NIC-NIIF son un modelo que toma como punto de partida el principio contable de la empresa en funcionamiento, y todas las normas que contiene van encaminadas a recoger el acontecer del día a día de los sujetos contables, con un fin fundamental: la elaboración y presentación de los estados financieros del ejercicio económico.

El Consejo Mexicano para la Investigación y Desarrollo de Normas de Información Financiera nos explica que utiliza el término "Normas de Información Financiera" en sustitución de los Principios de Contabilidad Generalmente Aceptados (PCGA), buscando enfatizar el carácter normativo y obligatorio de las norma de información financiera porque:

El vocablo principios sugiere grados de rigidez, permanencia y universalidad que no denotan la naturaleza dinámica de una de las NIF, las cuales se van adaptando a los cambios experimentados en el entorno bajo el cual operan las entidades. Significa que las NIF se van modificando, otras se eliminan, o bien se van emitiendo nuevas disposiciones; por lo tanto, no debe considerarse lo afirmado en ellas como fundamento inamovible (Comisión de Principios de Contabilidad del Instituto Mexicano de Contadores, 2006a). 
Tabla 1.Principios que pasan a ser postulados básicos en las normas internacionales de información financiera

\begin{tabular}{ll}
\hline PRINCIPIOS CONTABLES & POSTULADOS BÁSICOS \\
\hline Entidad & Entidad Económica \\
Empresa en Marcha & $\begin{array}{l}\text { Negocio en Marcha } \\
\text { Sustancia Económica }\end{array}$ \\
Hechos Económicos & \\
\hline Costo como Base de Valuación & Devengación Contable \\
$\begin{array}{l}\text { Devengado } \\
\text { Realización }\end{array}$ & \\
\hline Revelación Suficiente o Exposición & Requisitos de calidad \\
Materialidad o Importancia Relativa & \\
\hline $\begin{array}{l}\text { Equidad } \\
\text { Moneda Común Denominador }\end{array}$ & Integridad \\
Prudencia & \\
\hline Periodo Contable & Asociación de Costos y Gastos con Ingresos \\
Costo como Base de Valuación & Valuación \\
Consistencia o Uniformidad & Consistencia \\
Partida Doble & Dualidad Económica \\
\hline
\end{tabular}

Fuente: Tomado de Comisión de Principios de Contabilidad del Instituto Mexicano de Contadores, 2006b.

Sin embargo, el tema polémico del nuevo modelo es la aplicación del Valor Razonable.

Así, el desafío actual de la contabilidad es, por una parte, mantener su registro cronológico de las causas y efectos de los hechos económicos y, por la otra, reconocer las causas y efectos de la incertidumbre y del tiempo sobre los recursos y obligaciones de la entidad, actualizando los valores históricos o descontando los valores futuros para traerlos a valor presente, de manera de presentar sus estados financieros a valor razonable (Gómez y América, 2009, p. 2). 


\section{3 ¿Por qué resulta necesaria la contabilidad mundial?}

Por muchas razones obvias, cuando se observa en perspectiva el mercado global:

1. Para incrementar las ventas y las utilidades internacionales mediante el despliegue de ventajas comparativas $y / 0$ competitivas.

2. Facilita el ingreso a mercados crecientes y/o rápidamente emergentes.

3. Contribuye a la reducción de costos vía inversión extranjera directa y/o creación de subsidiarias, aliado con el capital nativo.

4. Para proteger los mercados nacionales de los competidores extranjeros.

5. Para proteger los mercados internacionales de los competidores locales.

6. Para incrementar la adquisición de tecnología y de conocimientos administrativos globales.

7. Facilita el comercio internacional de las importaciones y exportaciones.

8. Proyecta en el largo plazo el crecimiento de la empresa nacional en corporaciones multinacionales que, según definición de las Naciones Unidas, "son empresas que poseen o controlan las instalaciones de producción o de servicios fuera del país en el cual están basadas" (Organización de las Naciones Unidas [ONU], 1973, p. 23).

9. Ayuda a la inscripción en forma cruzada de sus acciones en bolsas de valores extranjeras.

10. Para reducir los costos de financiamiento de futuros proyectos de inversión por ampliación de planta en cualquier parte del mundo (Rugman y Hodggets, 1995).

\subsection{Enfoque}

Llegados a este nivel de análisis, queda clara la presencia de dos modelos dominantes de contabilidad que se usan en el mundo desarrollado en el manejo de la información internacional: 
a. El modelo europeo-continental. El principal objetivo de la contabilidad europea ha sido proteger a los acreedores, pues la tradicional forma de financiación de las empresas europeas ha sido la financiación ajena, sobre todo vinculada a entidades de crédito, que promueve un nivel débil de financiamiento externo de capital.

b. El modelo británico-estadounidense. El sistema contable de EE. UU. deriva del modelo anglosajón, y el componente financiero pesa más que el legal. Una consecuencia directa de ello es que nuestro sistema contable ha estado más ligado a normas fiscales, mientras que el norteamericano no. La normativa US GAAP es muy voluminosa, y está basada en normas e interpretaciones muy detalladas y pormenorizadas, donde hay un nivel fuerte de financiamiento externo de capital.

Sin embargo, el principal objetivo de la contabilidad norteamericana es ofrecer información a los inversores, pues su tradicional fuente de financiación son los mercados de valores. Está claramente orientada al inversor, bajo la idea de que si la empresa ha pedido fondos en el mercado, debe informar adecuadamente al que se los prestó, para que pueda decidir sus inversiones con base al riesgo. De ahí el enfoque de balance, la primacía de los flujos de caja y las importantes exigencias de información a los mercados. No se piensa de forma prioritaria en la Hacienda Pública, ni en los acreedores, de ahí que no se imponga necesariamente el método del coste histórico, sino el "fair value", ni se generan plusvalías ocultas derivadas de la aplicación estricta del criterio de prudencia. En este modelo no tienen cabida, por tanto, nuestras tradicionales provisiones "por si acaso".

Y si aún quedan dudas de la orientación final que tienen las Normas Internacionales, que priorizan la liquidez a la rentabilidad, veamos las ventajas que tiene la promoción del mercado de capitales:

1. Provee a las compañías la facilidad para conseguir el capital necesario para iniciar una inversión o negocio. 
2. Canaliza los ahorros de las economías domésticas hacia las necesidades de inversión de los distintos sectores económicos, dando como resultado un fuerte desarrollo económico y altos niveles de productividad.

3. Facilita el crecimiento de las empresas a través de fusiones, nuevas inversiones, expansión del mercado, operaciones todas ellas que necesitan de grandes sumas de capital proveniente de la institución bursátil.

4. Al ser las acciones un título de propiedad sobre una parte del capital de una empresa, las obliga a mejorar sus estándares de eficacia de la gerencia para satisfacer las demandas de estos accionistas, además de obligar a los gobiernos corporativos de las empresas a proporcionar información económico-financiera de la forma más transparente posible.

5. Crea oportunidades de inversión para los pequeños inversores.

6. Actúa como el barómetro de la economía, ya que la estabilidad económica se refleja en los precios de las acciones, los cuales actuarán manteniéndose o aumentando en el caso de bonanzas económicas. Sin embargo, en épocas de crisis financieras o recesión se produce un desplome general de todos los índices bursátiles.

\section{Resultados y discusión}

\subsection{Resultados}

Los escándalos financieros acaecidos en los Estados Unidos a finales de 2001 levantaron una serie de críticas que demandaban a reguladores y emisores de normas contables la revisión y mejora de las mismas.

En 2002 el Congreso de los Estados Unidos emitió la Ley Sarbanes-Oxley, orientada a restaurar la confianza del inversor. La ley no tenía como único objetivo la identificación de los "malos actores" y de sus "malas prácticas", sino que llegó a cuestionarse si las normas contables no eran las responsables de tales conductas. 
El Congreso de EE. UU. ordenó a la SEC llevar a cabo una revisión de los enfoques existentes para emitir normas de contabilidad, con el fin de determinar el camino más adecuado para cumplir con lo establecido por la citada ley (Reglas o principios generales).

En el año 2001, el IASC se convirtió en la Fundación IASCF y la labor de expedir Estándares Internacionales de Información Financiera pasó al IASB (International Accounting Standards Board). A partir del año 2001, la IASB inició el proceso para emitir los IFRS o NIIF, que sustituyen a las IAS o NIC, cambiando el enfoque de norma de contabilidad por Estándar de Información Financiera. Las IFRS o NIIF, que sustituyen a las IAS o NIC, no son solo cambio de nombre, se pasa de un esquema de preparación y presentación de E/F, basado en el registro, a otro completamente diferente de medición y presentación de reportes sobre el desempeño financiero (Ramírez, 2004, p. 6).

Es el fin del conservadurismo y del costo como base de valuación para ingresar a los postulados básicos, cuya característica fundamental es "la adaptación a los cambios experimentados en el entorno" como la volatilidad de la economía mundial de la mano del "fair value" o "valor razonable" (International Accounting Standards Board [IASB], 2012)².

\subsection{Discusión}

¿El valor razonable contribuirá a mejorar el patrimonio de las empresas en el mundo?

¿Hemos claudicado los profesionales contables ante la eliminación del costo histórico?

2 El precio que sería recibido por vender un activo o pagado por transferir un pasivo en una transacción ordenada entre participantes del mercado en la fecha de la medición (es decir, un precio de salida). Esa definición de valor razonable enfatiza que es una medición basada en el mercado, no una medida específica de una entidad (International Accounting Standards Board [IASB], 2012). 
¿Es mejor el enfoque probabilístico al de prudencia para evaluar los activos y pasivos contingentes?

¿No nos obliga la dualidad contable a priorizar lo económico-financiero a solamente la liquidez?

Es aún corto el tiempo para emitir una respuesta rotunda a las preguntas que flotan en el ambiente profesional contable. Pero sí sabemos con certeza las secuelas que nos deja el modelo internacional del IASB:

- Práctica desaparición del resultado extraordinario.

- Las acciones propias se presentan como menos patrimonio.

- Las acciones preferenciales, si tienen fecha de devolución, se presentan como pasivos.

- El LIFO , prohibido.

- Los activos no corrientes disponibles para la venta se presentan dentro del activo corriente.

- En general, los gastos de constitución se considerarán gasto del ejercicio.

- Posibilidad de actualizar a valores razonables el activo fijo, intangibles y propiedades de inversión; el deterioro del valor obliga a aplicar Valor Razonable.

- Costos de desmantelamiento de activos fijos.

- El Fondo de Comercio o Goodwill deja de amortizarse.

- Provisiones con base al criterio de probabilidad, no con base al criterio de prudencia.

- Obligatoriedad de evaluar a valor razonable los instrumentos financieros y activos biológicos.

3 Last In, First Out. Método de valuación de inventarios "Último en entrar, primero en salir". 


\section{Conclusiones}

1. Se demostró que la diversidad contable produce diferencias significativas en la valuación de los ingresos y el patrimonio en la presentación de estados financieros entre países, como el caso de la Daimler-Benz, que reportó utilidad aplicando los principios de contabilidad alemanes, pero una pérdida con los estadounidenses. Se evidencia que son normas de alta calidad, orientadas al inversor, priorizan lo financiero a lo económico, son provenientes del consenso alcanzado por diferentes corrientes teóricas desarrolladas en las últimas décadas, validadas por la práctica de los negocios y cuya finalidad es reflejar la esencia económica de las operaciones y presentar una imagen fiel de la situación financiera de la empresa.

2. Al analizar los dos modelos dominantes de contabilidad: el europeo-continental y el británico-estadounidense, encontramos que el primero protegía el interés de los acreedores, en el segundo el componente financiero pesa más que el legal, donde se financian las inversiones en el mercado de valores, por tanto es más importante ofrecer información a los inversionistas para generar trabajo, mejorar el nivel de vida de las personas y promover ahorro e inversión.

3. En el contexto académico, promovamos los "Postulados Básicos", en reemplazo de los principios del siglo pasado, que son los siguientes:
a. Entidad Económica;
b. Negocio en Marcha;
c. Sustancia Económica;
d. Devengación Contable;
e. Integridad;
f. Asociación de Costos y Gastos con Ingresos;
g. Valuación;
h. Consistencia y
i. Dualidad Económica. 


\section{Recomendaciones}

a. En el Perú, para mejorar la transparencia, la comparabilidad de la información reportada, expandir las oportunidades para todos los inversionistas y promover el crecimiento económico vía asignación de capital más eficiente, la Ley 29720 (2011) en su artículo $5^{\circ}$ promueve las emisiones de valores mobiliarios y fortalece el mercado de capitales, establece que las sociedades que se encuentran fuera de la supervisión de la Superintendencia del Mercado de Valores, SMV, deben presentar estados financieros auditados, preparados conforme a las NIIF de acuerdo con los plazos y disposiciones de la SMV. Las empresas afectas son alrededor de 10.000 y estarán obligadas a practicar el principio de transparencia. Noticia positiva para los contadores peruanos.

b. La Federación de Colegios de Contadores Públicos del Perú, integrante de la Federación Internacional de Contabilidad, IFAC, está poniendo en práctica las NIIF. La Resolución SMV No. 011-2012-SMV/01 establece las siguientes disposiciones transitorias para los estados financieros auditados:

c. Primera. Por el año 2012 corresponde presentar estados financieros auditados a las entidades cuyos ingresos o activos totales al cierre de 2012 superen los 30,000 UIT, es decir, 108 millones de soles (en US\$ 41 ` 550,000). A partir de 2013 dicho límite se reduce a 3,000 UIT (en US\$ 4 ` 155,000); y,

d. Segunda. Las entidades comprendidas en el alcance del párrafo anterior podrán preparar, en el año 2012, estados financieros (los que son objeto de auditoría financiera externa) con base en las NIIF oficializadas por el Consejo Normativo de Contabilidad, CNC, o con base en las NIIF vigentes internacionalmente. Para fines del año 2013, los estados financieros deberán prepararse observándolas íntegramente.

5. La difusión de la normatividad del Modelo IFRS genera nuevos retos para Contadores y Gerentes Financieros:

En el aspecto tributario: Aplicación del Valor de Mercado a operaciones de compra-venta para contribuyentes en general. A la aplicación de Precios de Transferencia a Valor de Mercado en actividades entre vinculadas. 
En el aspecto contable: Abandono de la vieja contabilidad asentada en el pasado (Costo Histórico). Mudanza a la nueva contabilidad basada en el valor razonable (fair value) en el mercado de las NIIF.

En el aspecto financiero: Medición de los Activos y Pasivos a Valor Razonable y registrarlos en libros en un ambiente de globalización, comercio electrónico e instrumentos y activos financieros.

- Por consiguiente, es de interés mundial proponer al IASB que emita una NIIF que consagre los nuevos postulados básicos como de uso obligatorio, al estilo de la NIIF 13, referido al Valor Razonable.

\section{Referencias bibliográficas}

Andersen Consulting. (2000). Mercado de Valores Tecnológicos. España, Madrid. Comisión de Principios de Contabilidad del Instituto Mexicano de Contadores. (2006a). Norma de Información Financiera NIF-A1, Estructura de las Normas de Información Financiera. . (2006b). Norma de Información Financiera NIF-A2, Postulados Básicos.

Doupnik, T. y Perera, H. (2007).Contabilidad Internacional. México: Editorial McGraw Hill/Interamericana Editores S.A. de C.V.

Gómez, N. \& América, E. (2009). Métodos de Valuación a Valor Razonables y su Auditoría. Chile, Santiago: Universidad de las Américas.

International Accounting Standards Board [IASB]. (2012). Norma internacional de Información Financiera IFRS 13, Medición del Valor Razonable. . (2006). Norma internacional de Información Financiera IFRS 21, Efecto de las Variaciones en los Tipos de Cambio de la moneda extranjera. .(2005). Norma internacional de Información Financiera IFRS 1, Presentación de Estados Financieros.

Ley 29720. (Junio de 2011). Recuperado el 31 de marzo de 2013 de http://www. congreso.gob.pe/ntley/Imagenes/Leyes/29720.pdf

Organización de las Naciones Unidas [ONU]. (1973). Multinational Corporations in World Development.

Pérez, J. (Junio de 2010). www.tecnicacontable.com. Recuperado el 31 de marzo de 2013 de Normas Internacionales de Información Financiera NIC/NIIF 
- Una verdadera revolución: Efectos contables de la convergencia financiera mundial: http://www.elcriterio.com/seccion_articulos/tecnicacontable_jorge_perez3.pdf

Ramírez, E. (2004). Bases y Fundamentos de la Contabilidad Financiera Internacional. Presentación en Salón Contable Universidad ICESI.

Rugman, A. y Hodggets, R. (1995). International Business: A Strategic Management Approach. Nueva York: Mc GrawHill.

Silva, B. y Azúa, D. (2006). Alcances sobre el Concepto de Valor Razonable. Chile: Escuela de Comercio - Pontificia Universidad Católica de Valparaíso.

Wikipedia. (s.f.). Crisis de 1929. Recuperado el 31 de marzo de 2013 de http:// es.wikipedia.org/wiki/Crisis_de_1929 